BULL. AUSTRAL. MATH. SOC.

$20 \mathrm{D} 10,20 \mathrm{D} 25$

VOL. 36 (1987) $475-483$

\title{
MODULAR FITTING FUNCTORS IN FINITE GROUPS
}

\author{
Peter Hauck and Reinhold Kienzle
}

\begin{abstract}
We consider Fitting classes for which the injectors in any finite solvable group are modular subgroups. It is shown that only normal Fitting classes have this property. In fact, we prove two more general results demonstrating that modular Fitting functors and submodular Fitting classes are normal.
\end{abstract}

\section{Introduction}

All groups considered in this paper are assumed to be finite. A class $X$ of groups (closed under isomorphisms) is called a Fitting class if it satisfies the following two properties:

(1) If $G$ is a group in $X, N$ a normal subgroup of $G$, then $N$ is in $x$.

(2) If $G$ is the product of two normal subgroups belonging to $X$, then $G$ is in $X$.

The relevance of Fitting classes lies in the theory of finite solvable groups and stems from the fact that each Fitting class $X$ gives rise to a distinguished conjugacy class of subgroups in any finite solvable group,

Received 30 January 1987.

Copyright Clearance Centre, Inc. Serial-fee code: 0004-9727/87 $\$ 2.00+0.00$. 
the so-called $X$-injectors. A subgroup $U$ of $G$ is called an $X$-injector if $U \cap N$ is maximal among all subgroups of $N$ contained in $\mathrm{X}$ (X-maximality) for all normal subgroups $N$ of $G$. The well-known result of Fischer, Gaschütz and Hartley ([3], Satz 1) states that every finite solvable group $G$ possesses $X$-injectors precisely when $X$ is a Fitting class, and in this case all $X$-injectors of $G$ are conjugate.

It follows from the definition that, for a given Fitting class $X$, every group $G$ has a unique largest normal subgroup contained in $X$, the $X$-radical $\operatorname{Rad}_{X}(G)$. Clearly, any $X$-injector of $G$ contains $\operatorname{Rad}_{X}(G)$.

The concepts of injector and radical have been generalized by Beidleman, Brewster and Hauck [1], [2] in the following way. Let $U$ be either the class $F$ of all finite groups or the class $S$ of all finite solvable groups.

A Fitting functor $f$ (in $U$ ) assigns to each group $G$ in $U$ a nonempty set $f(G)$ of subgroups of $G$ such that the following conditions are satisfied:

(1) If $\alpha$ is an isomorphism of $G$ onto $\alpha(G)$, then $f(\alpha(G))=$ $\{\alpha(X) \mid X \in f(G)\}$.

(2) If $N$ is a normal subgroup of $G$, then $f(N)=\{X \cap N \mid X \in f(G)\}$.

If $X$ is a Fitting class, then, denoting by $\operatorname{Inj}_{X}(G)$ the set of $X$-injectors of a given group $G$, it is not hard to see that $f=\operatorname{Rad}_{X}$ is a Fitting functor in $F$ and in $S$ and that $f=\operatorname{Inj}_{X}$ is a Fitting functor in $S$. In fact, these are examples of so-called conjugate Fitting functors, that is $f(G)$ consists of a single conjugacy class of subgroups for any group $G$ in the respective universe $U$.

Our main interest in this paper is in normal Fitting functors, that is those Fitting functors $f$ such that, for all groups $G$ in $u, f(G)$ consists only of normal subgroups of $G$.

It is an easy exercise to prove that a normal conjugate Fitting functor $f$ in $u=S$ or $F$ (that is $|f(G)|=1$ for all groups $G$ in 
4) is of the form $f=\operatorname{Rad}_{X}$ for some Fitting class $X([1], 3.10 . a)$. Considerably deeper is the statement that, in general, a normal Fitting functor is a union $f=u\left\{\operatorname{Rad}_{X_{i}} \mid i \in I\right\}$ of radical-functors for certain Fitting classes $x_{i}([1], 7.5)$.

The first purpose of the present paper is to prove that modular Fitting functors are normal (and hence of the form described above), where, of course, a Fitting functor $f$ in $U$ is called modular if, for any group $G$ in $U$, all members of $f(G)$ are modular subgroups of $G$. We recall that a subgroup $U$ of $G$ is modular if the following two properties are satisfied:

$$
\begin{aligned}
& \langle U, M\rangle \cap N=\langle M, U \cap N\rangle \text { whenever } M \leq N \leq G \text { and } \\
& \langle U, M\rangle \cap N=\langle U, M \cap N\rangle \text { whenever } U \leq N \leq G, M \leq G .
\end{aligned}
$$

Since modularity of subgroups is not a transitive relation, it is natural to consider the concept of a submodular subgroup. (The concept of "submodularity" has been introduced and investigated by I. Zimmermann (Freiburg) in a yet unpublished thesis.) Clearly, a subgroup $U$ of $G$ is termed submodular if there exists a chain $U=U_{0} \leq U_{1} \leq \ldots \leq U_{n}=G$ such that $U_{i}$ is modular in $U_{i+1}$ for $i=0, \ldots, n-1$.

If, for a given group $G, f_{s m}(G)$ is defined to be the set of all submodular subgroups of $G$, then it is obvious that $f_{s m}$ is a non-normal Fitting functor. Therefore, a result like the one mentioned above for modular Fitting functors does not hold for submodular Fitting functors. The same phenomenon occurs for subnormal Fitting functors; but in this case, it is rather easy to show that conjugate subnormal Fitting functors (or more generally, subnormal Fitting functors satisfying the Frattini argument; see $[1], 3.5)$ are normal.

Whether an analogous result holds for conjugate submodular Fitting functors, remains an open question. We have, however, a positive answer for the important case of injectors for Fitting classes in finite solvable groups. 


\section{Notation}

The group theoretical notation used in this paper is mainly standard.

$C_{n}$ stands for the cyclic group of order $n$ and $\mathbb{F}_{q}$ for the field with $q$ elements.

The proofs of the theorems depend on certain wreath product constructions. For groups $G$ and $H, G$ wr $B$ denotes the regular wreath product. If $K$ is a subgroup of $G$, then $K^{*}$ stands for the direct product of $|H|$ copies of $K$ in the base group of $G w r H$, and $K_{i}$ denotes the image of $K$ under the natural embedding into the $i$-th component of $K^{*}$. The projection from $G^{*}$ onto the $i$-th component is always denoted by $\pi_{i}$.

Finally, for a group $G$ with subgroup $H$, Core $G^{(H)}$ stands for the largest normal subgroup of $G$ contained in $B$ and $H^{G}$ for the smallest normal subgroup of $G$ containing $H$.

\section{The results}

THEOREM 1. Let $f$ be a modular Fitting functor in $U=F$ or $S$. Then $f$ is normal, that is there exists a family of Fitting classes $\left\{X_{i} \mid i \in I\right\}$ such that $\left.f(G)=U \operatorname{Rad}_{X_{i}}(G) \mid i \in I\right\}$ for every group $G$ in $U$.

THEOREM 2. Let $x$ be a Fitting class such that the $X$-injectors of any finite solvable group $G$ are submodular in $G$. Then $X$ is a normal Fitting class, that is $\operatorname{Rad}_{X}(G)$ is $X$-maximal in $G$ for all finite solvable groups $G$.

\section{The proofs}

Proof of Theorem 1. Suppose the theorem is false and let $G \in U$ be a group of minimal order such that there exists a non-normal $V \in f(G)$.

Let $K$ be a finite solvable group of nilpotent length at least 4 
and of order coprime to the order of $G$ and set $B=G$ wr $K \in U$. Choose $T \in f(B)$ such that $T \cap G_{1}=V_{1}$. We prove first that $T$ is contained in $G^{*}$.

Let $\pi$ be the set of prime divisors of the order of $K$. By the Schur-Zassenhaus theorem, $T$ has a Hall $\pi$-subgroup $Q$. By the same theorem, there exists some $x \in G^{*}$ such that $Q^{x} \leq K$. Note that $T^{x} \in f(H)$. If $Q \neq 1$, we may assume without loss of generality that $G_{2}^{q}=G_{1}$ for some $q \in Q$. It follows that $G_{1} \times G_{2}=\left\langle G_{2}, q\right\rangle \cap\left(G_{1} \times G_{2}\right)$ $=\left\langle G_{2}, T^{x}\right\rangle \cap\left(G_{1} \times G_{2}\right)=\left\langle G_{2}, T^{x} \cap\left(G_{1} \times G_{2}\right)\right\rangle$ by the modularity of $T^{x}$. Consequently, $\pi_{1}\left(T^{x} \cap\left(G_{1} \times G_{2}\right)\right)=G_{1}=\pi_{1}\left(T \cap\left(G_{1} \times G_{2}\right)\right)$. Using the fact that $T \cap G_{1}$ is normal in $\pi_{1}\left(T \cap G^{*}\right)$, we conclude that $V$ is normal in $G$, a contradiction. Hence $Q=1$.

This shows that $T \leq G^{*}$, whence $T^{H} \leq G^{*}$. We set $C=C_{H}\left(T^{H} /\right.$ Sore $\left._{H}(T)\right)$ and claim that $C$ is contained in $G^{*}$.

Assuming the contrary, the fact that $C$ is normal in $B$ and the Schur-Zassenhaus theorem imply the existence of some non-trivial element $k \in C \cap K$. Without loss of generality, $G_{1}{ }^{k}=G_{2}$. Let $g \in V$. Then $(g, 1, \ldots, 1) \in T$ and $(1, g, 1, \ldots, 1)=(g, 1, \ldots, 1)^{k} \in T^{H}$. Thus $\left(g^{-1}, g, 1, \ldots, 1\right) \in \operatorname{Core}_{H}(T)$ as $k \in C$. Then $\left(g^{-1} g^{y}, 1, \ldots, 1\right)=$ $\left(g^{-1}, g, 1, \ldots, 1\right)\left(g, g^{-1}, 1, \ldots, 1\right)^{(y, 1, \ldots, 1)} \in \operatorname{Core}_{H}(T) \leq T$ for any $y \in G$. Consequently, $[V, G] \leq \pi_{1}\left(T \cap G_{1}\right)=V$ that is $V$ is normal in $G$, a contradiction.

Therefore $C$ is contained in $G^{*}$ and, by the choice of $K, H / C$ has nilpotent length at least 4 . Since $T$ is modular in $H$, this contradicts Satz 5 of [8].

Thus $f$ is a normal Fitting functor. The structure of $f$ now follows from Korollar 7.5 of [1]. 
480

The following lemma is essential for the proof of Theorem 2 . In its statement we use the well-known fact that a group with a unique minimal normal subgroup possesses a faithful irreducible module over $\mathbb{F}_{p}, p$ a prime, if this minimal normal subgroup is not a $p$-group.

LEMMA. Let $p$ and $q$ be different primes, $A=C_{p}, B$ a faithful irreducible A-module over $I F_{q}, C$ a faithful irreducible $A B$-module over $I_{p}, D$ a faithful irreducible $A B C$-module over $I_{q}$, and set $H=A B C D$. (AII products are semidirect products with respect to the obvious action.) Then none of the groups $A B C, B C$, and $A C$ is submodular in $H$.

Proof. $A B C$ is a maximal subgroup of $H$, Core ${ }_{H}(A B C)=1$. Since $A B C$ is not nilpotent, it follows from Lemma 1 of [8] that $A B C$ is not modular and hence not submodular in $H$.

Now let $X$ stand for $B C$ or $A C$. Assume that $X$ is submodular in $H$. The only maximal subgroups of $H$ containing $X$ are $X D$ and all complements of $D$ in $B$ that contain $X$. Since $H$ is primitive, the latter ones are conjugate to $A B C([6], I I .3 .2)$ and thus not modular in $H$ by what we have proved above. Since in solvable groups maximal modular subgroups are maximal subgroups ([8], Lemma 1), there exists a chain $1=D_{0}<D_{1}<\ldots<D_{r}=D$ of $X$-invariant subgroups in $D$ such that $D_{i+1} / D_{i}$ is an irreducible $X$-module and $X D_{i}$ is a (maximal) modular subgroup of $X D_{i+1}$ for $i=0, \ldots, r-1$. We claim that at least one of the modules $D_{i+1} / D_{i}$ is not 1-dimensional. For otherwise, $X^{\prime}$ centralizes all modules $D_{i+1} / D_{i}$. Since $X$ is not abelian $(C$ is a faithful $A B$-module), $X^{\prime}$ is a non-trivial subgroup of $C$. Being a $p$-group centralizing a sexies of a $q$-group, $p \neq q, X^{\prime}$ centralizes $D$. This contradicts the fact that $D$ is a faithful $A B C$-module.

So there exists some $j, 0 \leq j \leq r-1$, such that $D_{j+1} / D_{j}$ is at least 2-dimensional. Thus $q^{2}$ divides the order of $X D_{j+1} /$ Core $_{X D_{j+1}}\left(X D_{j}\right)$. 
Functors in finite groups

Since $X D_{j}$ is maximal modular in $X D_{j+1}$, this contradicts Lemma 1 of [8].

Proof of Theorem 2. We may assume that $x \subseteq S$ (by replacing $x$ by $X \cap S$ if necessary) and $X \neq(1)$. Then $X$ contains $C_{r}$ for any prime $r$.

For otherwise, there exist primes $p$ and $q$ such that $c_{p} \in X$ and $C_{q} \notin X$. Taking $H$ to be the group of the Lemma, it follows that the $X$-injectors of $H$ are just the sylow $p$-subgroups of $H$ (see [4], section 3.3). Since $A C$ is not submodular in $H$, this is a contradiction.

We now show that $G^{2}$ wr $C_{p} \in X$ for all primes $r$ (where $G^{2}$ stands for $G \times G)$.

Assuming the contrary, there is a group $G$ in $X$ of minimal order such that there exists a prime $q$ with $G^{2}$ wr $C_{q} \notin X$. By the remark made above, $G \neq 1$. Let $N$ be a maximal normal subgroup of $G$, of index $p$ say ( $p$ a prime). Then $N \in X$ and thus $N^{2} w r C_{q} \in X$ and $N^{2}$ wr $C_{p} \in X$ by the minimal choice of $G$. It follows that $G^{2}$ wr $C_{p}$, being a product of the two subnormal subgroups $N^{2}$ wr $C_{p} \in X$ and $\left(G^{2}\right)^{*} \in X$, is contained in $X$. In particular, $p \neq q$.

Let $H$ be the group of the Lemma (with the same meaning of $p$ and $q$ as here) and set $K=G^{2}$ wr $H$.

Let $V$ be an $X$-injector of $K$. clearly, $\left(G^{2}\right) * \leq V$.

suppose there exists a non-trivial subgroup $D_{1}$ of $D$ such that $\left(G^{2}\right) * D_{1} \in X$. We may assume $D_{1}$ to be of order $q$. Since $\left(G^{2}\right) * D_{1} \cong G^{2 m}$ wr $C_{q}$, where $m$ is the index of $D_{1}$ in $H$, it follows from 5.11.a of [5] that $G^{2}$ wr $C_{q} \in X$, a contradiction.

we conclude that $V \cap\left(G^{2}\right) * D=\left(G^{2}\right) *$. 
since $G^{2}$ wr $C_{p} \in X, 5.1$ and $5.9 \mathrm{a}$ of [5] imply that $\left(G^{2}\right) * C \cong G^{2 n}$ wr $C$ ( $n$ is the index of $C$ in $H$ ) is contained in $X$. Consequently, by passing to a suitable conjugate of $V$, we may assume that $V \cap\left(G^{2}\right) * C D=\left(G^{2}\right) * C$.

If $V /\left(G^{2}\right) *$ is a $p$-group, then $V$ is conjugate to $\left(G^{2}\right) * A C$ since the latter group is isomorphic to $G^{2 Z}$ wr $A C \quad(2$ is the index of $A C$ in $H Y$ and thus contained in $X$ by 5.1 and 5.9 a of [5] again. otherwise, it follows easily from the fact that injectors cover or avoid chief-factors $([4]$, Lemma 1$)$ that $V$ is conjugate to $\left(G^{2}\right) * B C$ or to $\left(G^{2}\right) * A B C$. Since $V$ is submodular in $K, V /\left(G^{2}\right) *$ is submodular in $K /\left(G^{2}\right) *$. We conclude that one of the groups $A C, B C$, or $A B C$ is submodular in $B$. This contradicts the Lemma. We have proved that $G^{2}$ wr $C_{r} \in X$ for all $G \in X$ and all primes $r$. This implies that $X$ is a normal Fitting class by $5.11 b$ of [5] and the theorem of Makan [7].

\section{References}

[1] J.C. Beidleman, B. Brewster and P. Hauck, "Fittingfunktoren in endlichen auflösbaren Gruppen I", Math. Zeit. 182 (1983), 359-384.

[2] J.C. Beidleman, B. Brewster and P. Hauck, "Fitting functors in finite' soluble groups II", Math. Proc. Cambridge Phil. Soc. 101 (1987) 37-55.

[3] B. Fischer, W. Gaschütz and B. Hartley, "Injektoren endlicher auflösbarer Gruppen", Math. Zeit. 102 (1967), 337-339.

[4] B. Hartley, "On Fischer's dualization of formation theory", Proc. London Math. Soc. (3) 19 (1969), 193-207.

[5] P. Hauck, "Fittingklassen und Kranzprodukte", J. Algebra 59 (1979), 313-329.

[6] B. Huppert, Endliche Gruppen I, (Springer-Verlag, Berlin, Heidelberg, New York, 1967).

[7] A.R. Makan, "Fitting classes with the wreath product property are normal", J. London Math. Soc. (2) 8 (1974), 245-246.

[8] R. Schmidt, "Modulare Untergruppen endlicher Gruppen", IZZinois J. Math. 13 (1969), 358-377. 


\section{Functors in finite groups}

Mathematisches Institut

Albert - Ludwigs - Universitat

Albertstrasse $23 \mathrm{~b}$

D-7800 Freiburg i.Br.

Federal Republic of Germany 